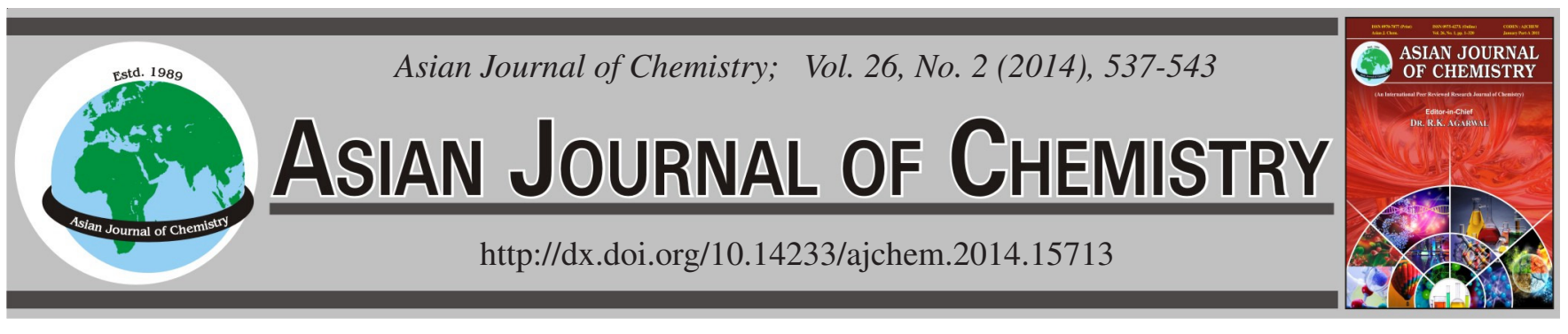

\title{
Preparation and Properties of Poly(vinyl alcohol) Fluorescence Fiber Acetalized with Glutaraldehyde
}

\author{
H.S. Yue, X.H. Deng ${ }^{*}$, Y. Sun, X.Q. Deng and Y. XIAO
}

State Key Laboratory of Hollow Fiber Membrane Materials and Processes, School of Material Science and Engineering, Tianjin Polytechnic University, Tianjin, P.R. China

*Corresponding author: Tel/Fax: +86 22 83955055; E-mail: dengxinhua0829@163.com; yhs198974@163.com

Received: 1 May 2013;

Accepted: 13 July 2013;

Published online: 15 January 2014;

AJC-14582

\begin{abstract}
Hydrophilic poly(vinyl alcohol) (PVA) fluorescence fiber was formed by dispersing oil soluble fluorescent pigment in PVA aqueous solution and wet spinning. The prepared fluorescence fiber was crosslinked with glutaraldehyde to improve the stability in hot water. Filament strength tester was used to determine the tensile properties of acetalized fiber. The crosslinked fluorescence fibers were observed by hot stage microscope and X-ray diffraction. Hot stage microscope observation revealed that the meltable PVA fluorescence fiber could be transformed into infusible by acetalization. The XRD results showed that the acetalization took place in the amorphous region of the fiber. The transformations of the crystal structures may happen if both the fluorescent pigment and the water get involved in the fiber but have nothing to do with the acetalization of the PVA fluorescence fiber. The fluorescence spectrum indicated a maximum absorption of the crosslinked fluorescence fiber is $373 \mathrm{~nm}$ and a maximum excitation wavelength is $494 \mathrm{~nm}$, thereby it demonstrated that the acetalization didn't damage the fluorescence nature of the fiber. As the acetalization took place in amorphous regionthe process didn't damage the PVA fluorescence fiber mechanical properties.
\end{abstract}

Keywords: Fluorescence fiber, Poly(vinyl alcohol), Glutaraldehyde, X-Ray diffraction, Fluorescence spectrum.

\section{INTRODUCTION}

Ultraviolet fluorescence fiber is widely used in anti-counterfeiting. It is colourless under visible-light but illustrates itself colours under UV-light. Preparations of fluorescence fiber have been reported in melt spinning method ${ }^{1-3}$ and melt complex spinning method ${ }^{4}$. UV phototropic anti-counterfeiting paper is made from paper pulp with fluorescence fiber. The paper turns to various colours from achromaticity under ultraviolet radiation.

Therefore, the fiber which can be mixed with paper pulp in any amount, should be hydrophilic. While fiber with poor moisture absorption is difficult to mix with paper pulp, poly(vinyl alcohol) (PVA) fiber which has good hydrophilicity can meet the processing requirements.

However, the preparation methods of the fluorescence fiber mentioned in those papers are not suitable to make PVA fluorescence fiber. Poly(vinyl alcohol) has large intermolecular force and pushes the melting temperature range up to around 225$230^{\circ} \mathrm{C}$, higher than its decomposition temperature $200-220^{\circ} \mathrm{C}$. It makes the poly(vinyl alcohol) melt-spinning unfeasible.

Poly(vinyl alcohol) fiber has to be made through wet spinning. The wet spinning process of poly(vinyl alcohol) is as follows: after poly(vinyl alcohol) aqueous solution was filtered and deaerated, the poly(vinyl alcohol) spinning solution is sent to the spinning plate by the spinning metering pump and then poly(vinyl alcohol) fibers are formed in coagulation bath. The poly(vinyl alcohol) fiber made via the method as described can dissolve in water, so it can not be used as clothes fiber.

The acetalization of poly(vinyl alcohol) fibers using other kinds of aldehyde were studied by Dorset ${ }^{5}$. He found that using benzaldehyde as crosslinking agent could lead to more flexible fiber than using formaldehyde. The acetalization between poly(vinyl alcohol) and formaldehyde or benzaldehyde occurred intramolecularly and made the fiber more draftability, but the poly(vinyl alcohol) not crosslinked. However, this kind of structure leads to larger interval between the molecules than a crosslinked network when swollen. Moreover, formaldehyde is poisonous and may pollute the environment.

Another cross-link agent of poly(vinyl alcohol), glutaraldehyde has been widely used in many research field. For example, Araujo et al. ${ }^{6}$ prepared poly(vinyl alcohol) membrane using glutaraldehyde as cross-link agent to prepare poly(vinyl alcohol)-glutaraldehyde network as a support for protein immobilization. Ramires and Milella ${ }^{7}$ studied membranes of 
poly(vinyl alcohol)-hyaluronic acid and poly(vinyl alcohol)gellan with different compositions by solution casting technique and crosslinked by exposure to the vapors of glutaraldehyde in an acid environment.

This paper describes the preparation of hydrophilicity poly (vinyl alcohol) fluorescence fiber by dispersing oil-soluble fluorescent pigment in PVA aqueous solution and then wet spinning to produce a colourless fluorescence fiber. However, after mixing with fluorescent pigments, the hot water resistance of PVA fluorescence fiber will decrease and the fiber will be able to dissolve in water above $70{ }^{\circ} \mathrm{C}$.

In this study, bi-functional glutaraldehyde as crosslink agent not only has less toxicity but also can crosslink the PVA molecules. As the fluorescent pigment has to be embedded into the fiber macromolecules, it is necessary to have the PVA intramolecularly crosslinked. The network will avoid the loss of fluorescence dye in the process of swelling. The goal of our work is to use glutaraldehyde as a crosslinking agent in making the fluorescence fiber and to study how the glutaraldehyde crosslinking agent and fluorescent pigment affect the structures and the characteristics of the PVA fluorescence fibers.

\section{EXPERIMENTAL}

Poly(vinyl alcohol) was purchased from Fujian Textile and Chemical Fiber Co. in China. The degree of polymerization and saponification were 1799 and 99 mol \%, respectively. The fluorescent pigment, a pale yellow powder was bought from Tianjin Yunxing Printing Ink Co. in China and used as received without further purification. Anhydrous $\mathrm{Na}_{2} \mathrm{SO}_{4}, \mathrm{H}_{2} \mathrm{SO}_{4}$ and a glutaraldehyde water solution ( $50 \%$ by weight) as the crosslink agent, all in reagent grade, were procured from Tianjin Tiantai Fine Chemicals Co. in China and used as received without further purification.

Preparation of PVA fluorescence fiber: Oil soluble fluorescent pigment emulsion (grain size $\leq 500 \mathrm{~nm}$ and $14 \mathrm{wt} \%$ PVA aqueous solution were mixed with a ratio of $0.5 \%$ as the spinning fluid. After filtering and de-airing, wet spinning was used to make the colourless and opacity fiber. The wet fiber was through the $\mathrm{Na}_{2} \mathrm{SO}_{4}$ aqueous solution in a coagulation bath, leading the dry fiber with a draft ratio 4 to 6 .

Acetalization reaction of PVA fluorescence fiber: A series of solutions, each containing $200 \mathrm{~mL}$ distilled water, $8 \mathrm{~mL}$ concentrated sulfuric acid ( $98 \%$ by weight), $30 \mathrm{~mL}$ ethyl alcohol, $12 \mathrm{~g}$ anhydrous $\mathrm{Na}_{2} \mathrm{SO}_{4}$ and glutaraldehyde with different concentration (2-8 wt \% relative to total weight of reaction solution), were prepared. The PVA fluorescence fiber was then dipped into the solution with a bath ratio of 1:150. As the fiber could be dissolved apparently at temperature above $70{ }^{\circ} \mathrm{C}$, the acetalization had to take place at $50-60{ }^{\circ} \mathrm{C}$. The reaction last 0.25 to $0.5 \mathrm{~h}$ at that temperature with a small tension force on it. The acetalized fibers were washed at room temperature with distilled water repeatedly to clean the residual chemicals and then dried at $120^{\circ} \mathrm{C}$.

Analytical methods and equipment: $\mathrm{X}$-Ray diffractions were conducted using D8 DISCOVER with GADDS (BRUKER AXS CO., US) X-ray diffraction instrument and Ni-filtered $\mathrm{CuK}_{\alpha}$ radiation $(\lambda=1.54178 \AA)$. All the tests were done at room temperature and under atmosphere pressure condition.
Hot-stage microscope photos were taken with BX51 (Olympus Co., Japan) industry microscope, equipped with an Olympusc-5050 Zoom digital camera, a JVC TK-C1481BEC colour video frequency vidicon and a LINKAM THMS 600 currency cold-hot stage controlled by temperature programming.

Tensile properties of PVA fluorescence fibers were measured using a tensile tester of TOM/5 model of Shinko Tsushin Kogyo (Japan) at $20^{\circ} \mathrm{C}$ and a relative humidity $50 \%$.

Fluorescence measurements were done with a SpexFluorolog-2 DM1B fluorimeter in the front-face geometry with $2.5 \mathrm{~mm}$ excitation and emission slits (band pass $=4.5 \mathrm{~nm}$ ). The excitation wavelengths for PVA fluorescence fibers were from 200 to $500 \mathrm{~nm}$.

Resistance to hot water test was conducted in the following steps: first, loosely roll some fiber bundles then dry it to a constant weight. Then put it into boiling water for $0.5 \mathrm{~h}$ and then take it out and dry it again to a constant weight followed by a calculation of the loss of weight rate. The process was repeated with the same sample for whatever times to have the procedure last for $3 \mathrm{~h}$. Each experiment performed was three times to get the average and the deviation.

Ultraviolet visible spectra were obtained with a Jin Dao UV-2550 UVVis spectrophotometer (Japan).

\section{RESULTS AND DISCUSSION}

Hot water resistance of PVA fluorescence fiber after acetalization: The PVA fluorescence fiber has to be acetalized before use for two reasons. First, the PVA fluorescence fiber, if un-acetalized, can be swelled and deformed easily in water at room temperature. Second, the fiber can dissolve quickly in hot water over $70^{\circ} \mathrm{C}$.

The acetalization changes the fiber in two ways. First, it could, at least partly, prevents the hydroxyl group from the attack of the water. Second, the acetalization can have PVA fluorescence fiber crosslinked. The combination is believed to work together to improve the hot water resistance of the PVA fluorescence fiber.

The improvement is observed in Fig.1, which used the weight loss as an indicator of the hot water resistance. After acetalization, the fiber experienced only $2.50-3.36 \%$ weight loss after 0.5 to $3 \mathrm{~h}$ in boiling water.

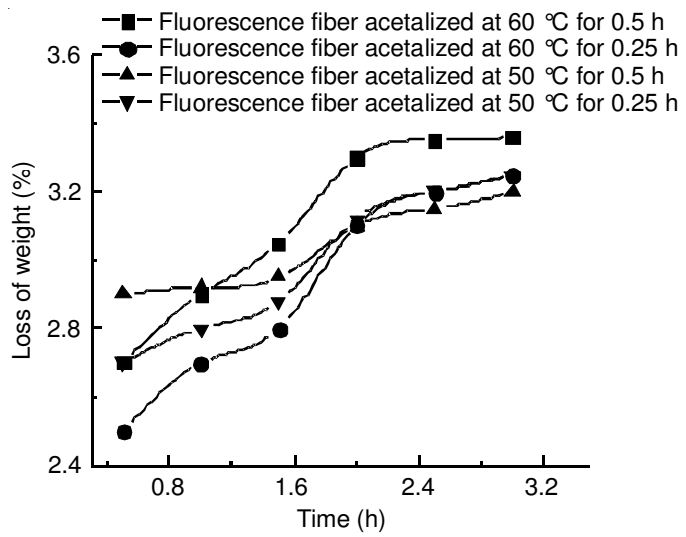

Fig. 1. Weight loss of PVA fluorescence fibers after acetalization with glutaraldehyde in boiling water (glutaraldehyde concentration 4.83 wt \%) 
It remained unknown if the pigment had been washed out or not. In this study, the PVA fluorescence fiber after acetalization was boiled in water for $3 \mathrm{~h}$ and then filtered. After the filtrate was cooled, its ultraviolet absorb characteristic was tested. Under the same conditions, acetalized PVA fiber was treated with boiling water, followed by filtering, so did the fluorescence powder and water mixture. As comparison, ultraviolet absorb characteristics of above three filtrates were tested. Results were shown in Fig. 2. It indicated that filtrate from acetalized PVA fiber had no absorption with the same diluted multiple. The filtrate from fluorescence powder had visible absorption at 200-365 $\mu \mathrm{m}$ although it didn't dissolve into water. But the filtrate from the acetalized PVA fluorescence fiber only had a weak absorption which demonstrated PVA fluorescence fiber forming crosslink network structure and only a little pigment had been washed out.

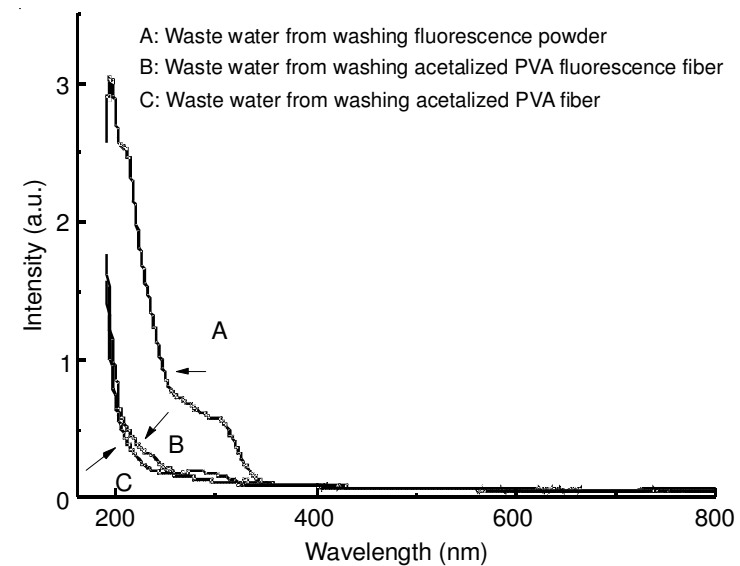

Fig. 2. Ultraviolet absorption spectra of the waster water from washing

Effects of acetalization conditions on mechanical behaviour of PVA fluorescence fiber: The mechanical behaviour tested in the work included break strength, elongation at break and the initial modulus. Filament strength tester was used to determine the break strength of the PVA fluorescence fibers which acetalized with glutaraldehyde of different concentrations. Fig. 3 showed the dependence of the breaking strength of the acetalized fiber on the glutaraldehyde's concentrations. All the test fibers had the highest breaking strength in 4.83 wt \% glutaraldehyde's concentration.

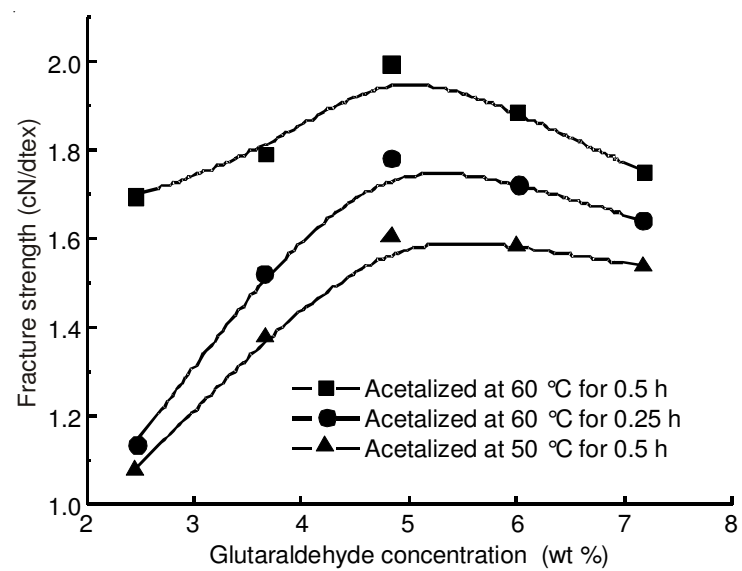

Fig. 3. Effects of glutaraldehyde concentration on fracture strength of fluorescence fibers acetalized with glutaraldehyde (titer: 12.79 dtex; sulphuric acid concentration 4.28 wt \%)
The reaction temperature is more significant than the reaction time in the acetalization. The break strength of fibers acetalized at $60{ }^{\circ} \mathrm{C}$ was higher than the ones made at $50{ }^{\circ} \mathrm{C}$.

Our fluorescence fibers are weaker than the commercial vinylon's short fibers, which usually possess break strength around 4.0-4.4 cN/dtex ${ }^{8}$. The break strength of fluorescence fibers before acetalization was $1.917 \mathrm{cN} / \mathrm{dtex}$ according to our test. After acetalization, the break strength of fluorescence fibers became 1.995, 1.781 and $1.605 \mathrm{cN} / \mathrm{dtex}$, respectively as shown in the three highest points in Fig. 3.

The data also indicates that acetalization at $60^{\circ} \mathrm{C}$ for $1 \mathrm{~h}$ seems to have not negatively impacted the mechanical behaviour even though sulfate has been used in the process.

There could be three reasons behind the behaviour. First, the fluorescent pigments added may negatively influence the fiber's capability of crystallizing and lead to a more disordered microstructure structure. Second, the draft ratio of our tested fluorescence fiber reached only 4 , compared to 8 to 10 with typical industrial product. Third, the vinylon short fiber was treated with formaldehyde rather than glutaraldehyde used in this work. Glutaraldehyde may reduce the interaction among molecules.

From Figs. 4 and 5 we could see that the elongation at break of acetalized PVA fluorescence fiber had a trend of decreasing along with extending reaction time and increasing reaction temperature and an increasing tendency with the initial modulus. This was because that the crosslink density of PVA macromolecules increased with rise of acetalization reaction time and prolong of reaction temperature in the experiment, which made relative displacement of chain segments among macromolecules much more difficult.

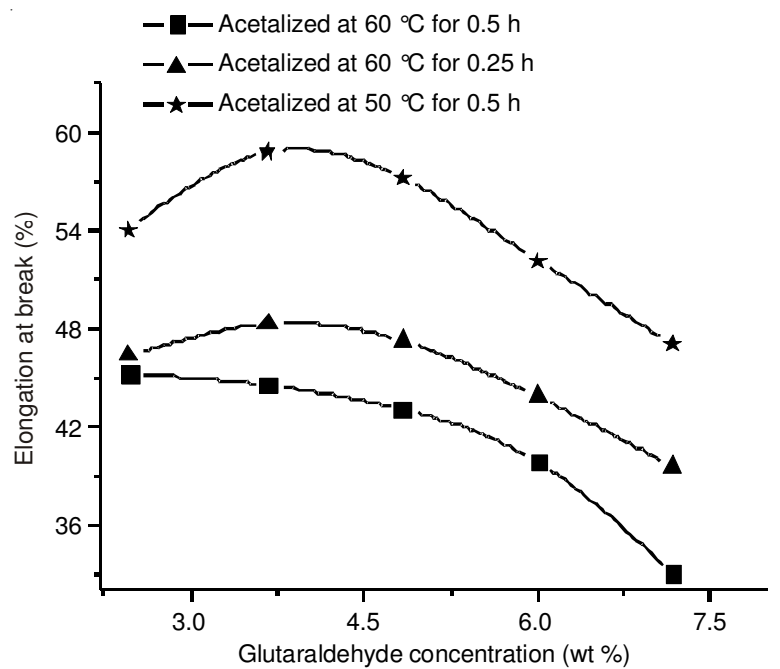

Fig. 4. Effects of glutaraldehyde concentration on elongation at break of fluorescence fibers acetalized with glutaraldehyde (titer: 12.79 dtex; sulphuric acid concentration 4.28 wt \%)

The initial modulus of all samples was the highest in 4.83 wt \% glutaraldehyde's concentration which was consistent with Fig. 3. The initial modulus of vinylon's short fiber by normal wet spinning was $22-62 \mathrm{cN} / \mathrm{dtex}^{8}$. Most experimental data in Fig. 5 were in these ranges.

The initial modulus of PVA fluorescence fiber before acetalization was $60.49 \mathrm{cN} / \mathrm{dtex}$ according to our test. Comparing 


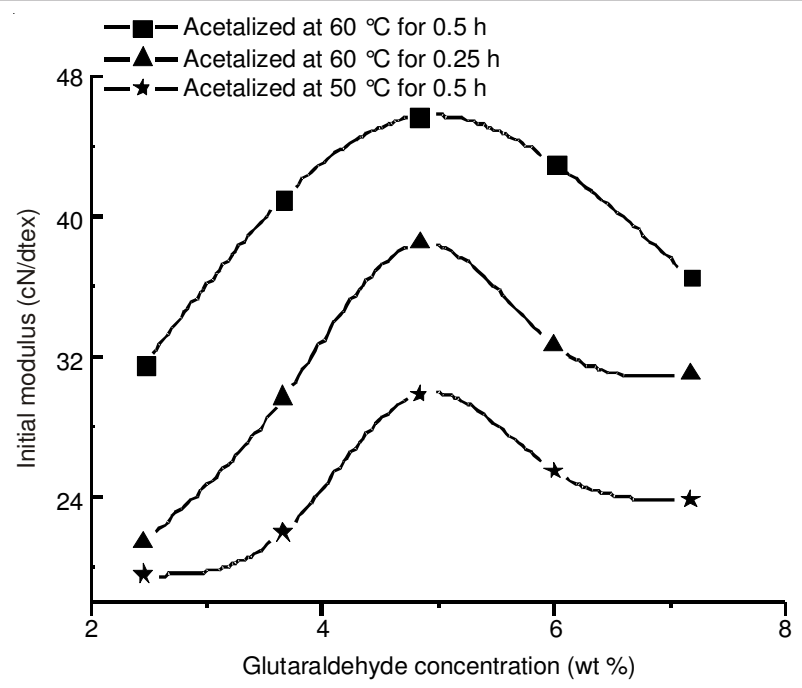

Fig. 5. Eeffects of glutaraldehyde concentration on initial modulus of fluorescence fibers acetalized with glutaraldehyde (titer: 12.79 dtexsulphuric acid concentration 4.28 wt \%)

with the initial modulus of PVA fluorescence fiber after acetalization, we found that acet alization could decrease the initial modulus of fiber.

The elongation at break of fluorescence fiber before acetalization was $29 \%$ according to our test, while the vinylon's normal wet spinning short fiber ${ }^{8}$ had $12-26 \%$. All the elongation at break of PVA fluorescence fiber after acetalization was larger than that of the upper limit of normal wet spinning vinylon's short fiber.

It's because that the fiber was winded loosely during acetalization process, resulting in chain segments disorientation in amorphous region. Also, normal wet spinning vinylon's short fiber was treated with formaldehyde, but our PVA fluorescence fiber was acetalized by glutaraldehyde. The flexible crosslink chain segments, which were equivalent to a plasticizer in the PVA fluorescence fiber, made it easy for the molecular chain segments to move and improved the polymer flexibility.

Influential factors on crystalline structures of PVA fluorescence fiber: Whether or not the acetalization process changed the crystalline structure of PVA fluorescence fiber was investigated by X-ray diffraction. For all the acetalized PVA fluorescence fibers samples in this test including the posterior samples, acetalization took place under the conditions: glutaraldehyde concentration of $4.83 \mathrm{wt} \%$, sulphuric acid concentration of $4.28 \mathrm{wt} \%, 60^{\circ} \mathrm{C}$ and $0.5 \mathrm{~h}$.

Fig. 6 was a group of equator X-ray diffraction graphs for PVA fluorescence fibers and Fig. 7 was the ones for unacetalized pure PVA fibers.

The comparison of Figs. 6a with 7a gave us an opportunity to see the fluorescent pigment effect on crystalline structure of the fiber. The two graphs looked similar, indicating that the fluorescent pigment inside fiber did not change the crystalline structure of fiber.

Fig. 6a-b were the X-ray diffractogram for the unacetalized and acetalized PVA fluorescence fibers. The main diffraction peaks were both in $19.5^{\circ}$. However, the two diffraction peaks at 31.95 and $33.9^{\circ}$ were observed in Fig. 6a without acetalization but not found in Fig. $6 \mathrm{~b}$ with acetalization. In addition,
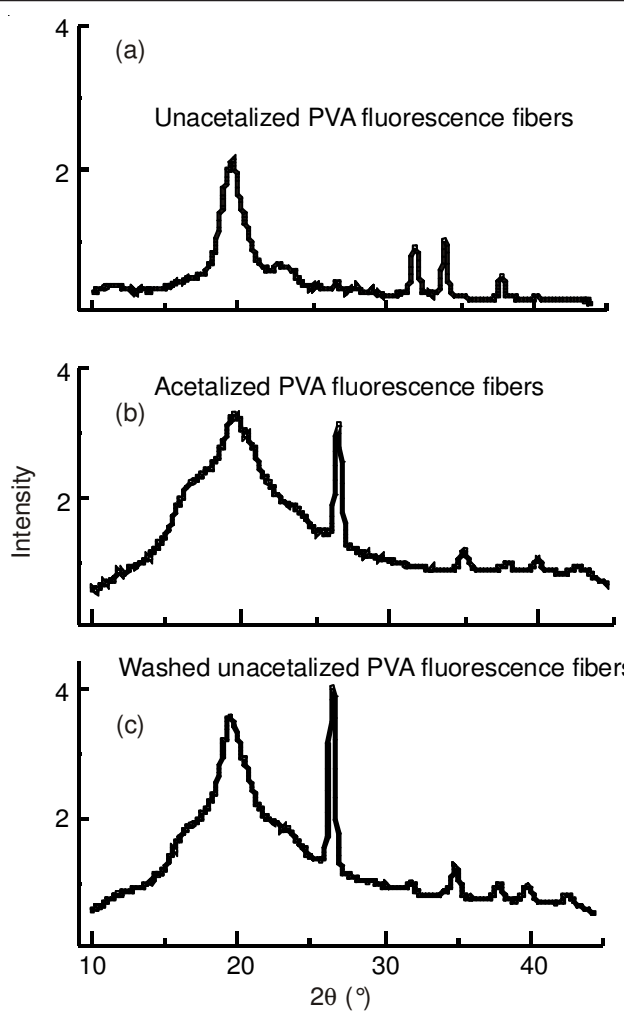

Fig. 6. X-Ray diffractograms of PVA fluorescence fibers

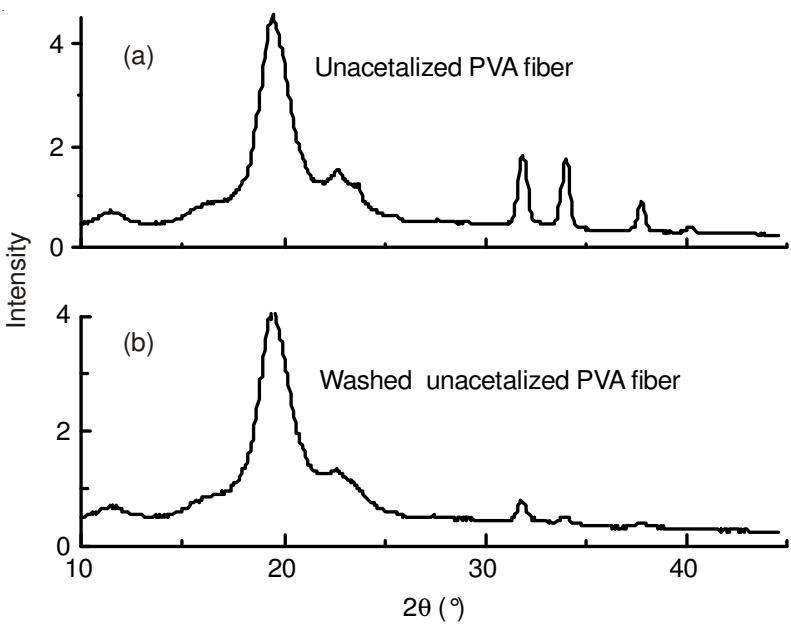

Fig. 7. X-Ray diffractograms of unacetalized PVA fibers

a strong new diffraction peak and three small new diffraction peaks appeared at $26.35,34.97,39.93$ and $42.41^{\circ}$ respectively in Fig. 6 b.

The change of the crystalline structure may not be necessary to be caused by the acetalization. Fig. 6c showed that the the X-ray diffractogram of fluorescence fiber only water washed but never unacetalized, we could see the same diffraction pattern as in Fig. 6 b.

The change of crystalline structure, however, was not caused only by the osmotic diffusion of water into the fiber. By comparing the two X-ray diffraction graphs of unacetalized PVA fiber unwashed (Fig. 7b) and washed (Fig. 7c), we found that the diffraction peaks remained the same.

The results showed that the coexistence of the fluorescent pigment and water inside fiber was the main factor that made the crystalline structure change of PVA fluorescence fiber. 


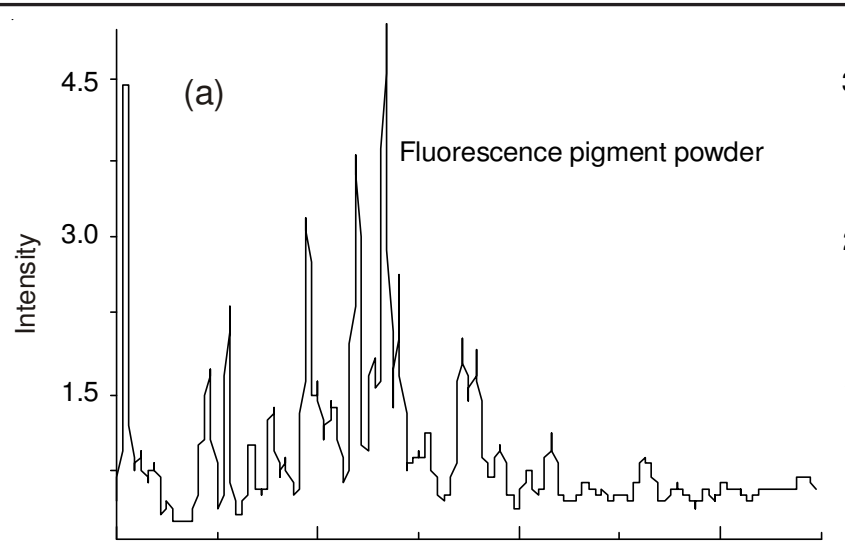

(b)
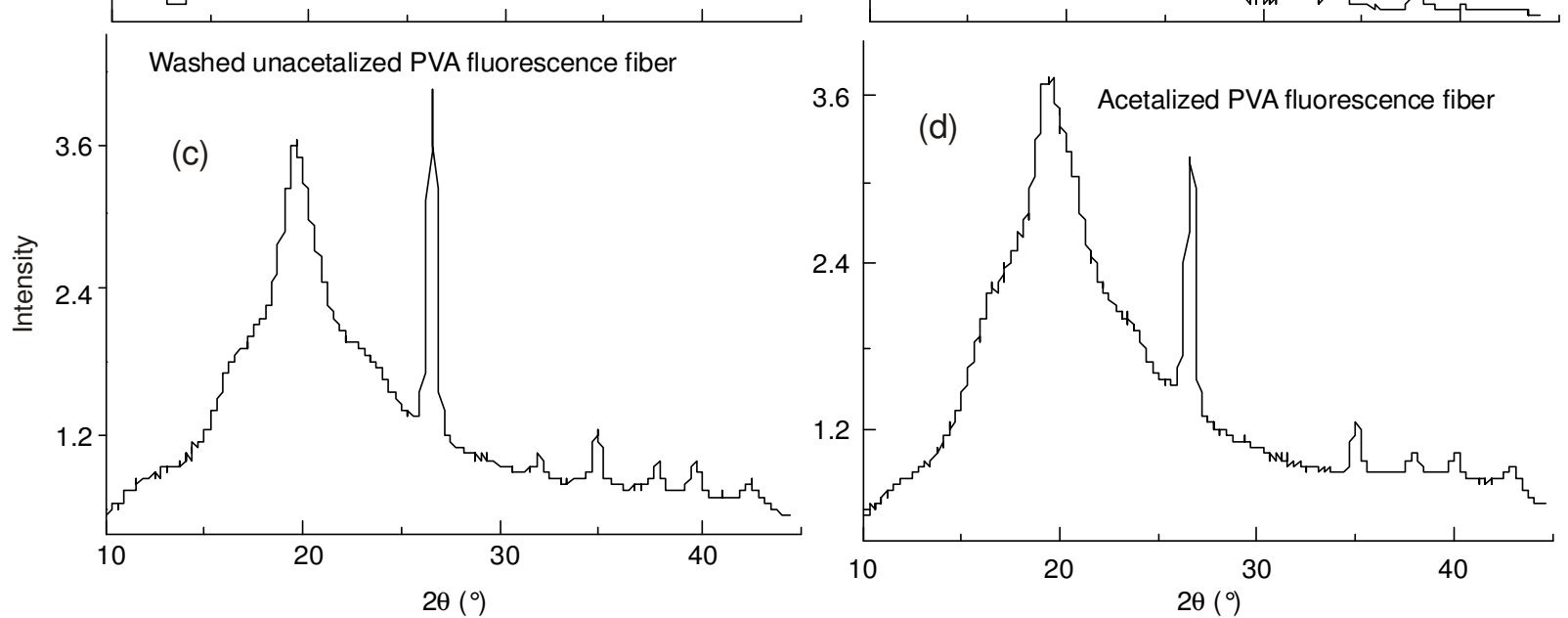

Fig. 8. X-Ray diffractograms of fluorescent pigment and fluorescence fibers

Again, the acetalization was not a factor that changed the crystalline structure.

Crystalline state of fluorescent pigment in PVA fluorescence fiber: The crystalline state of fluorescent pigment in PVA fluorescence fiber was observed by X-ray diffraction. Fig. 8 showed the X-ray diffraction spectrograms of fluorescent pigment and PVA fluorescence fibers, unacetalized, acetalized or washed.

Fig. 8 showed that fluorescent pigment powder showed sharp peaks of crystalline structure. However, the same peaks didn't show up in the PVA fluorescence fibers, unacetalized, acetalized or washed. This might be owing to the low content of fluorescent pigment. The weak diffraction might have been covered by the diffuse-light from the amorphous region in the PVA.

In order to prove the above speculation, the mixed solution of PVA and fluorescence pigment was prepared according to the ratio of the fiber to make the membrane. After preparing the membrane on the glass and drying at room temperature the membrane was investigated by X-ray diffraction at the same condition. In Fig. 9, the diffraction peak of the fluorescence pigment wasn't found in the case of containing fluorescence pigment, which indicated the diffraction peak was covered by the diffuse-light from the amorphous region in the PVA because of the low content of fluorescent pigment in the fiber.

Thermal stability of PVA fluorescence fiber without or with acetalization: Thermal stability of PVA fluorescence fiber without or with acetalization was measured by a hot-stage microscope. Fig. 10 showed the image (50X) of the unacetalized

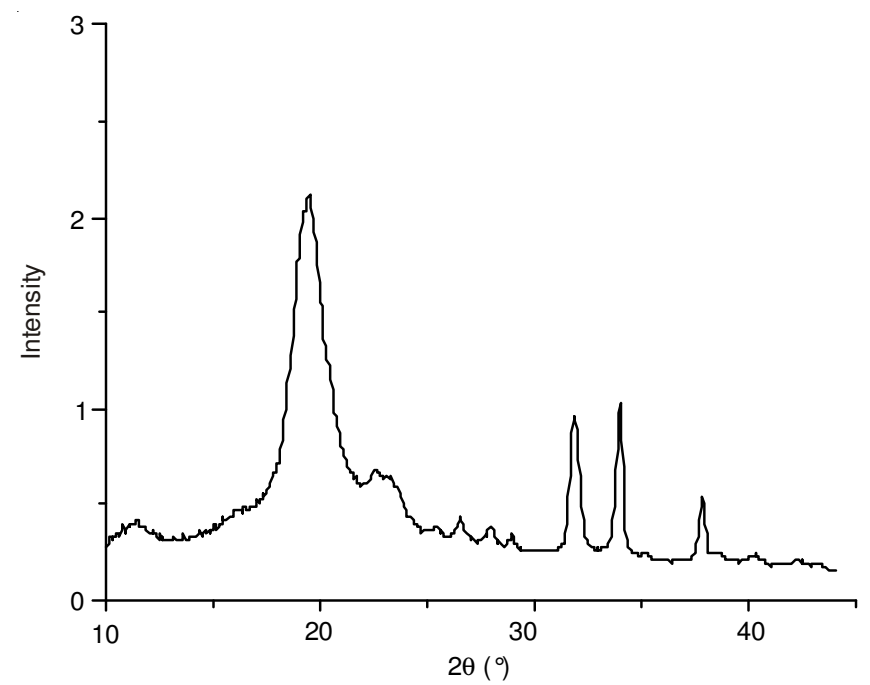

Fig. 9. X-Ray diffractograms of mixed membrane (fluorescent pigment and PVA)

PVA fluorescence fiber with a hot-stage microscope. The PVA fluorescence fiber began softening and dehydrating at about 200-230 ${ }^{\circ} \mathrm{C}$. Many particle-like dots, which were believed to be water drops from the dehydrating, could be observed around the fibers (Fig. 10b-c). It melted at about $248^{\circ} \mathrm{C}$ (Fig. 10d). When temperature cooled down to $162^{\circ} \mathrm{C}$, many small particles were observed in the cooled fused PVA polymer (Fig. 10e), which were proved to be crystal particles with polarized light.

Fig. 11 showed the image (50X) of acetalized PVA fluorescence fiber with the hot-stage microscope. The fiber dehydrated 


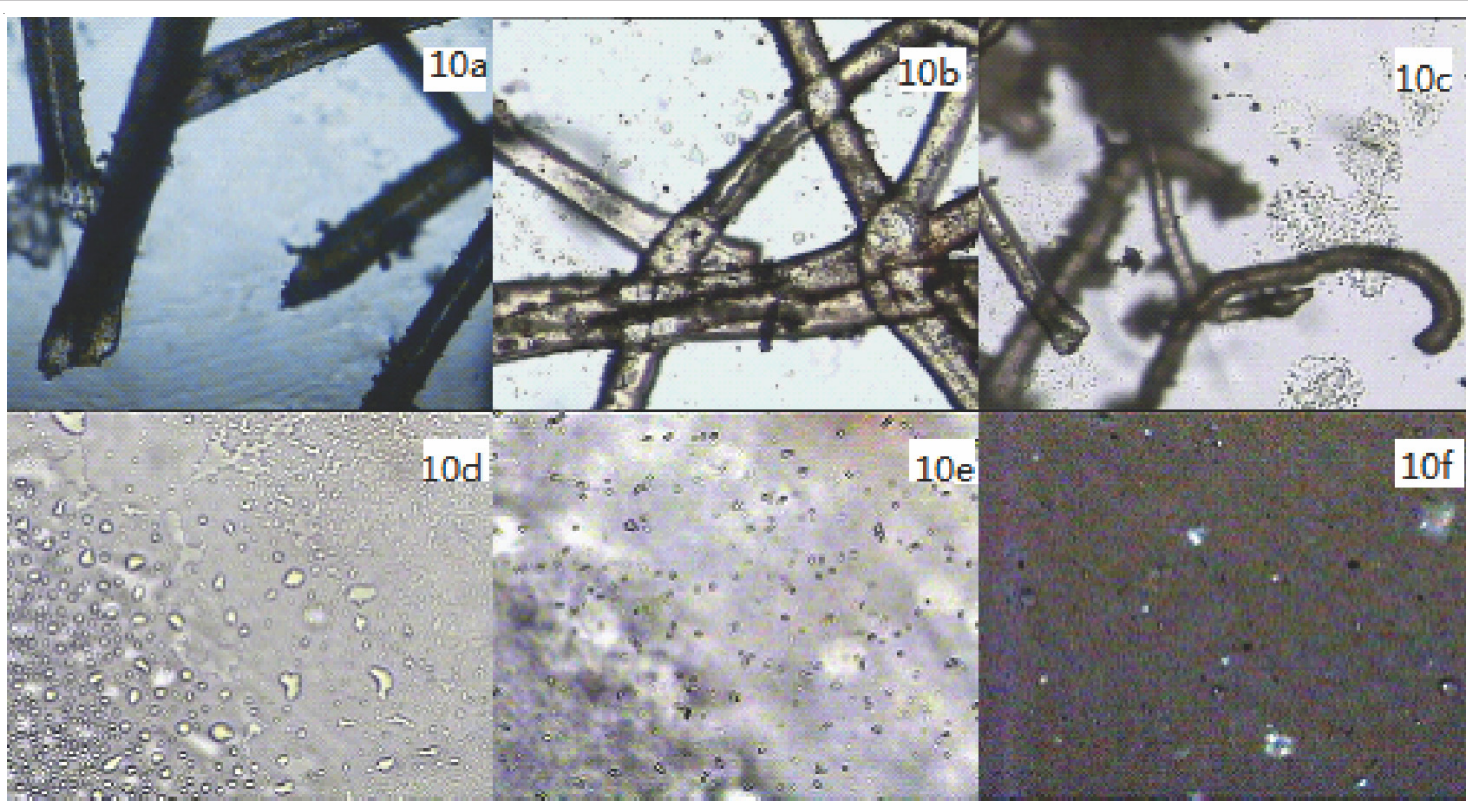

Fig. 10. Hot-stage microscope photos (50x) of unacetalized PVA fluorescence fiber under different temperatures a: $28^{\circ} \mathrm{C}$ b: $205^{\circ} \mathrm{C}$ c: $223^{\circ} \mathrm{C}$ d: $248^{\circ} \mathrm{C}$ e: $162{ }^{\circ} \mathrm{C} \mathrm{f:} 162{ }^{\circ} \mathrm{C}$ (polarized light)

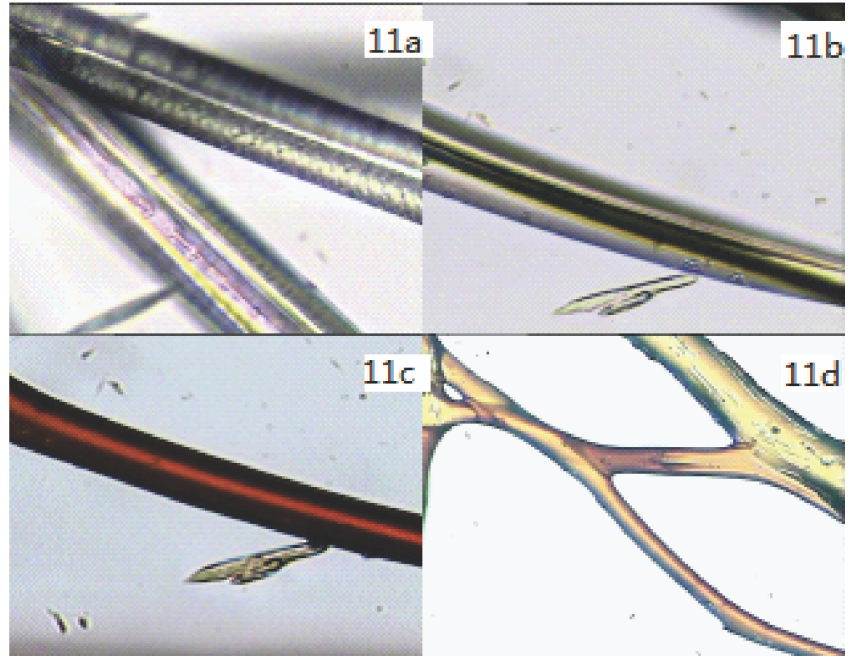

Fig. 11. Hot-stage microscope photos (50x) of acetalized PVA fluorescence fiber under different temperatures a: $202{ }^{\circ} \mathrm{C} ;$ b: $243^{\circ} \mathrm{C}$; c: $268^{\circ} \mathrm{C}$; d: $320{ }^{\circ} \mathrm{C}$

at $202{ }^{\circ} \mathrm{C}$ (Fig. 11a) with some minute liquid drops on the fiber surface. The fiber did not melt at about $243^{\circ} \mathrm{C}$ (Fig. 11b). It may be because of the acetalization had removed some of the hydroxyl groups on the PVA chains, the amount of the water drops were observed to be less than the one without acetalization (Fig. 10c). At $268^{\circ} \mathrm{C}$, the fiber became yellow and brown (Fig. 11c). The melting of the fiber was not observed up to it becoming dark in colour, which may indicate that the fiber had experienced a thermal deformation or degradation (Fig. 11d).

Apparently, the acetalization could improve the thermal stability of the PVA fluorescence fiber.

Fluorescence properties of PVA fluorescence fiber with acetalization: Fluorescence propertie of acetalized PVA fluorescence fiber were mensurated by a fluorimeter. Fig. 12 showed the excitation spectrum of the acetalized PVA fluorescence fiber. The maximum absorption wavelength is located

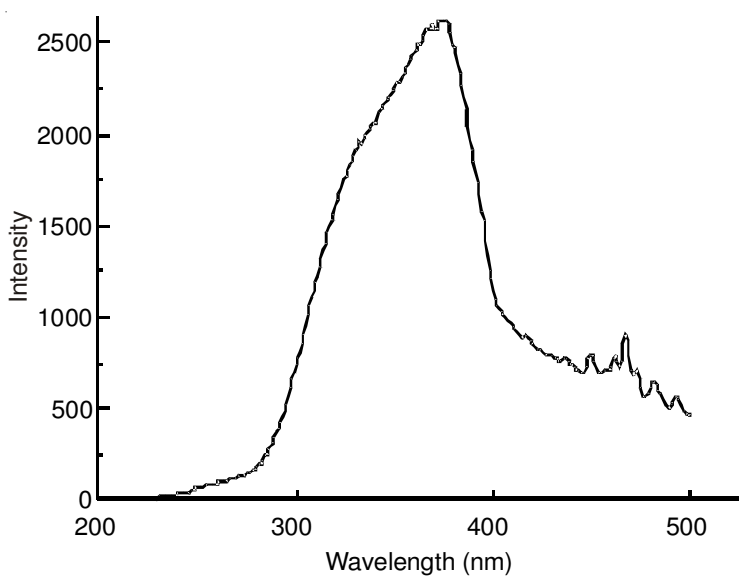

Fig. 12. Excitation spectrum of acetalized PVA fluorescence fiber

at $373 \mathrm{~nm}$. This wavelength was selected as excitation wavelength to test the emitted spectrogram of acetalized PVA fluorescence fiber.

Fig. 13 showed the emission spectrum of acetalized PVA fluorescence fiber. The results indicated that the fluorescence behaviour was there and apparent even with acetalization.

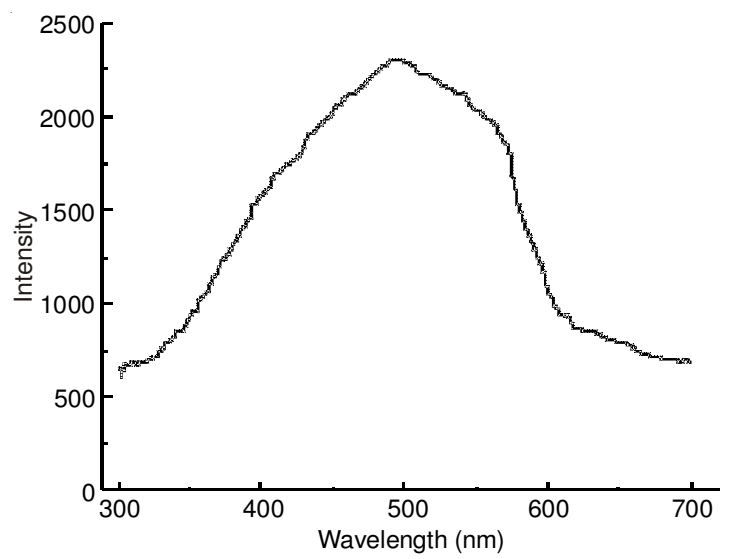

Fig. 13. Emission spectrum of acetalized PVA fluorescence fiber 
The fibers possessing long-wave fluorescence effect could be used as UV photochromic fibers. The fiber's emission spectrogram was broad and the wavelength of its strongest emitted light was located in $494 \mathrm{~nm}$, that the fiber looks green under UV light.

Comparing Fig. 11 with Fig. 12, we could find that the acetalized PVA fluorescence fiber not only contained stroke fluorescence, but, the fluorescence's wavelength was longer than excitation light's. It also included resonance fluorescence, which had the same wavelength as the excitation light. It was caused by some crystal existing in the fiber.

Theoretically, the fluorescence emission spectra and absorption spectra of fluorescent substance have mirrorimage symmetry. However, a deviation was observed in our test. There were several possible reasons for the deviation. First, it might partially be resonance fluorescence phenomena caused by PVA microcrystalline. Second, fluorescence spectrophotometer had higher accuracy and precision in the measurement of a dilute solution than in a concentrated solution. The system in our experiment to measure the acetalized PVA fluorescence fiber was solid state fibers, which was containing abundant microcrystalline PVA. It was equivalent to a concentrated solution. The system could possibly produce types of complexes, from combinations of the fluorescent substance molecules of excited and ground states or the PVA and excited fluorescent substance molecules, or even the fluorescent substance molecules themselves in the ground state. Again, the part of the emission was actually absorbed by the fluorescence fibers themselves, causing the decrease of fluorescence intensity in the fluorescence spectra ${ }^{9}$.

Effects of stretch ratio on acetalized PVA fluorescence fiber's crystallization: Effects of stretch ratio on acetalized PVA fluorescence fiber's crystallization were observed by X-ray diffraction. Fig. 14 showed the X-ray diffraction graphs of the fluorescence fibers after acetalization with different draft ratios. The drafting process was conducted at $190-200{ }^{\circ} \mathrm{C}$ and the intensity of equator scanning diffraction peak was enhanced with the increase of the draft ratio. The result indicated that tensile force was propitious to the orientation and arrangement of the PVA segments to form microcrystalline. Meridian scanning diffraction graph showed that the orientation degree of PVA fluorescence fiber from a larger draft ratio was higher.

\section{Conclusion}

Hydrophilic PVA fluorescence fiber could be formed by wet spinning, using the blend of the PVA aqueous solution and oil soluble fluorescent pigment. In the process, glutaraldehyde could be used as a crosslink agent to replaced formaldehyde. The acetalization not only caused no damage to the fluorescence nature of the fiber, but also made it unable to melt and to dissolve even in hot water. The acetalization did not change the crystalline structure of PVA fluorescence fiber. The crystalline
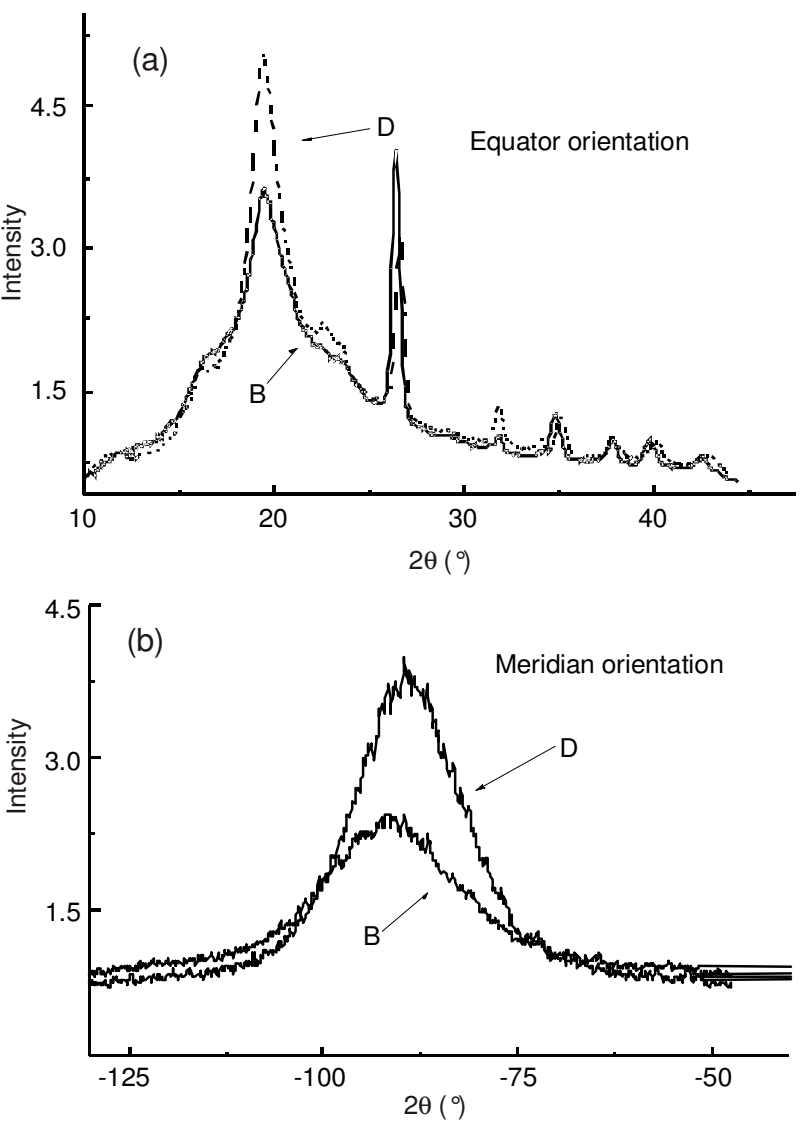

Fig. 14. X-Ray diffraction graphs of acetalized PVA fluorescence fibers with different drafting times; B: drafting 4 times; D: drafting 6 times

structure can only be changed through the coexistence of the inner fluorescent pigment and the water as a plasticizing agent. As the acetalization took place in amorphous region the process still left the PVA fluorescence fiber with good mechanical properties.

\section{REFERENCES}

1. R.H. Geisel, Method of Providing Luminescence to Fibrous Materials, US Patent 5674437 (1997).

2. W. Owens, Process for Producing Phosphorescent Yarn and Yarn Produced by the Process, US Patent 5321069 (1994).

3. L.E. Doane Jr., Toy Doll Construction with Phosphorescent Hair Fibers, US Patent 4781647 (1988).

4. Y. Shimizu, K. Ogasawara, K. Sakakura, A. Ogura and D. Goto, High Luminance Luminous Fiber and Process for Producing the Same, US Patent 6162539 (2000).

5. Dorset, Text. Manuf., 82, 636 (1956).

6. A.M. Araujo, M.T. Neves Jr. and W.M. Azevedo, Protein Immobil. Biotechnol. Techniq., 11, 67 (1997).

7. PP.A. Ramires and E. Milella, J. Mater. Sci. Mater. Med., 13, 119 (2002).

8. D. Jizhen, Z. Yaoming and C. Xueying, Synthetic Fiber Production Tectonics, China Textile Press, Beijing, edn. 2 (1994).

9. C. Guozhen, H. Xianzhi and X. Jingou, Fluorescence Analysis, China Science Press, Beijing, edn. 2 (1990). 